\title{
Achieving the Theoretical Depairing Current Limit in Superconducting Nanomesh Films
}

\author{
Ke $\mathrm{Xu},{ }^{\dagger}$ Peigen Cao, and James R. Heath* \\ Kavli Nanoscience Institute and Division of Chemistry and Chemical Engineering, California Institute of Technology, \\ MC 127-72, Pasadena, California 91125
}

\begin{abstract}
We show the theoretical depairing current limit can be achieved in a robust fashion in highly ordered superconductor nanomesh films having spatial periodicities smaller than both the superconducting coherence length and the magnetic penetration depth. For a niobium nanomesh film with $34 \mathrm{~nm}$ spatial periodicity, the experimental critical current density is enhanced by more than 17 times over the continuous film and is in good agreement with the depairing limit over the entire measured temperature range. The nanomesh superconductors are also less susceptible to thermal fluctuations when compared to nanowire superconductors. $T_{\mathrm{c}}$ values similar to the bulk film are achieved, and the nanomeshes are capable of retaining superconductivity to higher fields relative to the bulk. In addition, periodic oscillations in $T_{\mathrm{c}}$ are observed as a function of field, reflecting the highly ordered nanomesh structure.
\end{abstract}

KEYWORDS Superconductors, nanomaterials, electronic materials, nanofabrication

$\mathrm{T}$ he highest dissipationless current (supercurrent) a superconductor can carry is described by the depairing mechanism: ${ }^{1}$ when the kinetic energy associated with the supercurrent exceeds the condensation energy (e.g., the binding energy of Cooper pairs), superconductivity vanishes. However, the experimental $J_{\mathrm{c}}$ (critical current $I_{\mathrm{C}}$ divided by the cross section area) of bulk superconductors and superconductor films is typically more than 1 order of magnitude lower ${ }^{2,3}$ than this theoretical limit, the depairing current density $\left(J_{\mathrm{dp}}\right)$. Improved $J_{\mathrm{c}}$ has been obtained through patterning superconductors at micrometer and submicrometer scales, ${ }^{4-8}$ but the depairing limit is still not reached, and significantly increased current densities are only evident close to the critical temperature $\left(T_{\mathrm{c}}\right)$.

The reasons behind the large discrepancy between the experimental $J_{c}$ and the theoretical $J_{\mathrm{dp}}$ are 2 -fold. First, transverse dimensions larger than the magnetic penetration depth $\lambda$ lead to the piling-up of currents at the surfaces and/ or edges of the superconductor due to the Meissner effect. ${ }^{1}$ Second, for transverse dimensions larger than the superconducting coherence length $\xi$, vortices nucleate within the superconductor at high currents, the motion of which leads to dissipation and thus destruction of superconductivity. ${ }^{9}$ Superconductor wires having diameter smaller than both $\xi$ and $\lambda$ can, in principle, overcome both these limitations. Because both $\xi$ and $\lambda$ are large ${ }^{1}$ for temperature $(T)$ close to $T_{\mathrm{c}}$, early studies ${ }^{10}$ indicated that $J_{\mathrm{dp}}$ can be achieved in micrometer-size filaments for $T \sim T_{\mathrm{c}}$. However, in this regime $J_{\mathrm{c}}$ is much lower than its low- $T$ limit. For $T<\sim 0.9 T_{\mathrm{c}}$, both $\xi$ and $\lambda$ quickly reach their low- $T$ limits of $\sim 50 \mathrm{~nm}$, and

*Corresponding author, heath@caltech.edu.

† Current address: Department of Chemistry and Chemical Biology, Harvard University, 12 Oxford St., Cambridge, MA 02138.

Received for review: 07/23/2010

Published on Web: 08/25/2010
$J_{c}$ falls far below $J_{\text {dp. }}{ }^{10}$ Nanofabrication advances have permitted the exploration of superconductor nanowires ${ }^{1-18}$ (NWs) with transverse dimensions smaller than the low- $T$ limits of $\xi$ and $\lambda$. However, in this regime, NWs are quasione-dimensional, meaning that only one pathway is available for supercurrent passage. Consequently, supercurrents in NWs are readily disrupted by transient, local resistive "phase-slip" events due to thermal and quantum fluctuations. ${ }^{13}$ As a result, superconductor NWs are characterized by large residual resistance ${ }^{11-18}$ for $T \ll T_{\mathrm{C}}$, and the measured $J_{c}$ is much less than the $J_{\mathrm{dp}}$ of corresponding bulk materials. ${ }^{15,19,20}$

Here we report on a novel film structure that harnesses the advantages of NWs while circumventing the fluctuation effects characteristic of quasi-1D superconductors. This is achieved by building a highly ordered two-dimensional nanomesh film (Figure 1) with characteristic length scales well below both $\xi$ and $\lambda$. In such structures, the currentcarrying components are effectively NWs, so supercurrents over the cross section of each NW are uniform, and vortices cannot form within the NWs. Meanwhile, the small dimensions of the nanomesh lattice provide alternative pathways for supercurrents within distances that are well below the superconducting coherence length $\xi$. This means that the supercurrents can avoid transient local resistive events caused by thermal fluctuations. As a result, a large enhancement of $J_{\mathrm{c}}$ is observed, and the $J_{\mathrm{dp}}$ limit is achieved over a wide temperature range.

$\mathrm{Nb}$ nanomesh films were prepared using superlattice nanowire pattern transfer (SNAP), ${ }^{21,22}$ which translates the atomic control over the layer thicknesses within a thin film superlattice into control over the width and spacing of NWs. Briefly, two sets of Pt SNAP NWs were sequentially deposited onto a $\mathrm{SiO}_{2}$-Coated superconductor (Nb) thin film (thickness $=11 \mathrm{~nm}$ ) to form a crossbar structured etch mask (Figure 

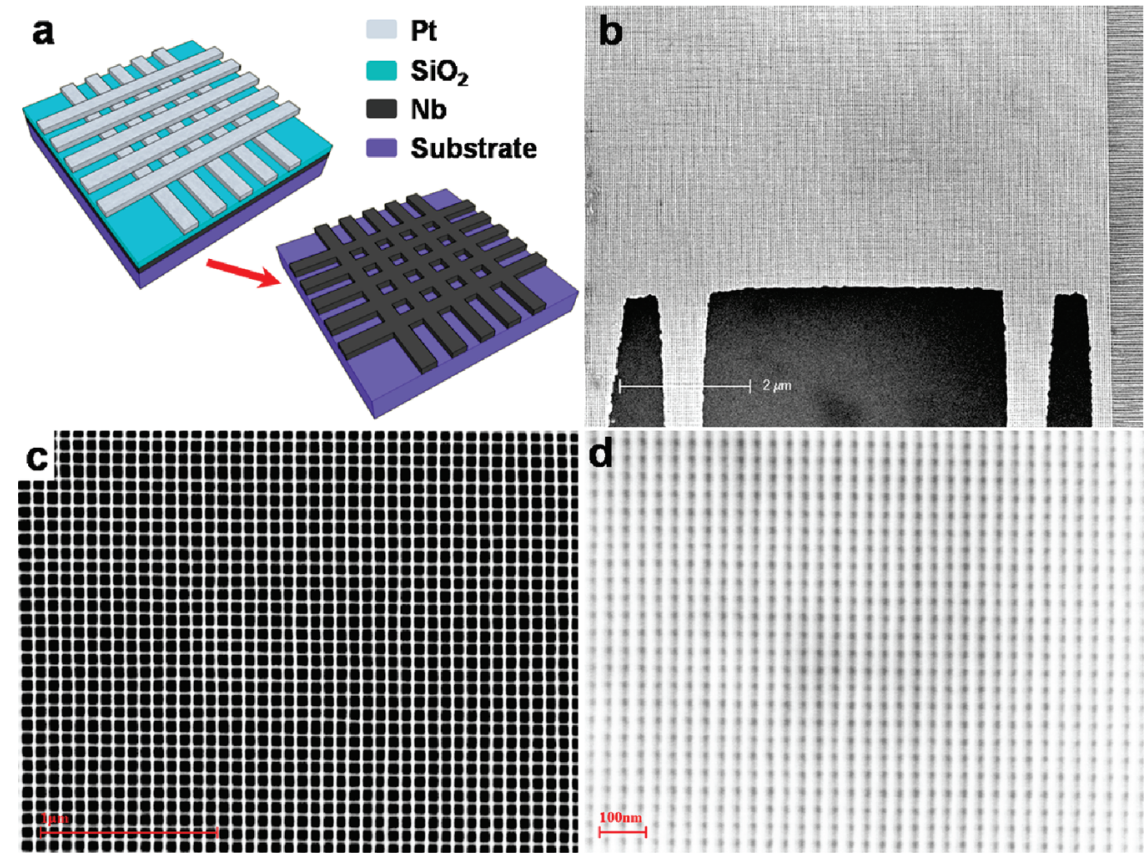

FIGURE 1. Fabrication of ultra-high-density superconductor nanomesh structures. (a) Illustration of the fabrication process. A Pt nanowire crossbar structure is formed on top of a $\mathrm{SiO}_{2}$-coated $\mathrm{Nb}$ thin film, providing an etch mask for forming a Nb nanomesh film. (b-d) Scanning electron micrographs of the nanomesh films investigated in this study. (b) Low-magnification image of a nanomesh device (34 $\mathrm{nm}$ pitch), showing two voltage probes patterned from the same Nb nanomesh (scale bar $2 \mu \mathrm{m}$ ). (c) High-magnification image of a Nb nanomesh patterned at $75 \mathrm{~nm}$ pitch, with $15 \mathrm{~nm}$ wide grid lines (scale bar $1 \mu \mathrm{m}$ ). (d) High-magnification image of a $34 \mathrm{~nm}$ pitch Nb nanomesh with $16 \mathrm{~nm}$ wide grid lines (scale bar $100 \mathrm{~nm}$ ). The $\mathrm{Nb}$ film thickness is $11 \mathrm{~nm}$ for both structures.

1a). Highly directional dry etching converted the Nb film into the nanomesh films shown in Figure $1 b-d$. The nanomesh devices were patterned to be $\sim 5 \mu \mathrm{m} \times \sim 5 \mu \mathrm{m}$ in area, with voltage probes made out of the same $\mathrm{Nb}$ nanomesh ${ }^{23}$ for four-point measurements (Figure $1 \mathrm{~b}$ and Supplementary Figure 1 in the Supporting Information). See Supporting Information for detailed methods. To interrogate dimensional effects, we prepared different pitch nanomesh films (34 and $75 \mathrm{~nm}$ ) to bracket $\xi$ of $\mathrm{Nb}(\sim 40 \mathrm{~nm})$. The widths of the nanomesh grid lines were similar (16 and $15 \mathrm{~nm}$ ) for both films. The sizes of the square voids in the nanomesh films are thus 18 and $60 \mathrm{~nm}$, respectively. The electrical properties of the nanomesh films were characterized and compared with a $10 \mu \mathrm{m}$ wide continuous thin film device, which was patterned from the same $11 \mathrm{~nm}$ thick starting $\mathrm{Nb}$ film as the nanomesh films.

Figure 2 a provides the measured temperature-dependent resistance $(R)$ of different devices at varied magnetic fields. Remarkably, at zero field, the $R-T$ behavior of the $34 \mathrm{~nm}$ pitch nanomesh (henceforth referred to as "34 nm nanomesh") is similar to that of the film. This contrasts with the $R-T$ behavior of individual NWs, ${ }^{1-18}$ which is characterized by significantly reduced $T_{\mathrm{c}}$, a severely broadened transition temperature width, and large residual resistance for $T \ll T_{\text {c }}$. This result demonstrates that nanomesh films patterned at pitches smaller than $\xi$ are not susceptible to thermal fluctuations, as supercurrents can readily find alternative pathways through interconnecting NWs when fluctuation-induced local resistance occurs. The larger pitch
$75 \mathrm{~nm}$ nanomesh exhibits a modest reduction of $T_{\mathrm{C}}$ and a slightly widened transition temperature width due to fluctuations. Meanwhile, in the presence of perpendicular magnetic fields $(H)$, the nanomesh films retain superconductivity to higher fields: at $H=1 \mathrm{~T}$, both nanomesh films have $T_{\mathrm{C}}$ higher than the film. At $H=2 \mathrm{~T}$, complete superconductivity is not achieved in the bulk film at base temperature $(1.7 \mathrm{~K})$, while it is achieved in nanomesh films at $T>3 \mathrm{~K}$. This is likely because the grid wires within the nanomesh have cross sections smaller than $\xi$ and $\lambda$, consistent with previous studies $^{17,24}$ on individual NWs.

The influence of $H$ is also clearly seen in the $T_{\mathrm{c}}-H$ data (Figure 2b) summarized from multiple $R-T$ curves collected at closely spaced $H$ (Supplementary Figure 2 in the Supporting Information). Due to the finite transition temperature width, a resistance criterion is used here to define $T_{\mathrm{c}}$, i.e., $T_{\mathrm{c}}$ is defined as the temperature at which the measured resistance is $50 \%$ of the normal state resistance, $R_{\mathrm{N}}$. Enhanced $T_{\mathrm{C}}$ relative to the film is observed for the $34 \mathrm{~nm}$ nanomesh for all $H$, while the $75 \mathrm{~nm}$ nanomesh has reduced $T_{\mathrm{C}}$ at low $H$ due to the larger pitch, consistent with previous discussions. In addition, on top of the general decreasing trend of $T_{\mathrm{c}}$ for increasing $H$, noticeable oscillating features are observed at certain fields for both nanomesh films.

Figure $2 \mathrm{C}$ presents the $T_{\mathrm{C}}$ oscillations of the $75 \mathrm{~nm}$ nanomesh obtained from $T_{\mathrm{C}}-H$ curves after subtracting a smooth, parabolic $T_{\mathrm{C}}-H$ trend. When $0.95 R_{\mathrm{N}}$ is used as the criterion for determining $T_{\mathrm{c}}$, four clear, ordered oscillation periods are observed, with peaks appearing at integral 

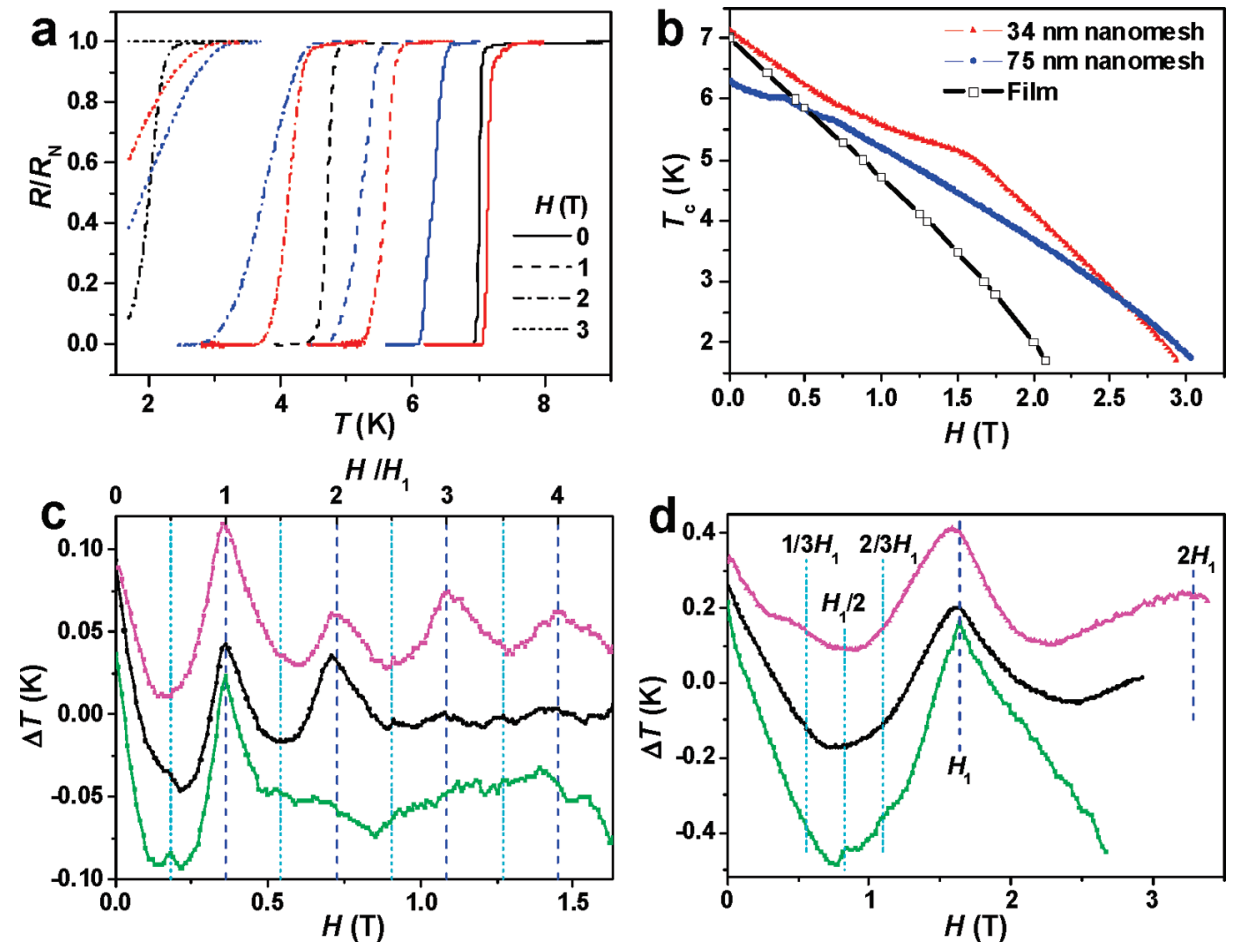

FIGURE 2. Temperature-dependent electrical resistance of the Nb nanomesh films and corresponding thin film. (a) Temperature-dependent resistance $(R)$ of different devices measured at applied (perpendicular) magnetic fields $(H)$ of $0,1,2$, and $3 \mathrm{~T}$ : red, $34 \mathrm{~nm}$ pitch nanomesh; blue, $75 \mathrm{~nm}$ pitch nanomesh; black, thin film device. The resistance is normalized to the respective normal-state resistance $\left(R_{\mathrm{N}}\right)$ for each device. (b) Magnetic field-dependence of $T_{\mathrm{c}}$ for the three devices, as defined by the temperature at which $R=0.5 R_{\mathrm{N}}$. (c) The $T_{\mathrm{c}}$ oscillations of the $75 \mathrm{~nm}$ pitch nanomesh as a function of $H$, obtained from the $T_{\mathrm{c}}-H$ curve after subtracting a smooth, parabolic $T_{\mathrm{c}}-H$ trend. (d) The $T_{\mathrm{c}}$ oscillations of the $34 \mathrm{~nm}$ pitch nanomesh as a function of $H$. For both (c) and (d), pink, black, and green curves are obtained with the criteria $R=0.95 R_{\mathrm{N}}, 0.5 R_{\mathrm{N}}$, and $0.05 R_{\mathrm{N}}$, respectively.

multiples of the period, $H_{1}=0.362 \mathrm{~T}$. When multiplied by the square of the $75 \mathrm{~nm}$ pitch, this value corresponds to the field at which the magnetic flux through each unit cell (loop) is $2.04 \times 10^{-15} \mathrm{~Wb}$, a value that is in good agreement with the magnetic flux quantum, $\Phi_{0}=h / 2 e=2.068 \times 10^{-15} \mathrm{~Wb}$. This indicates that the oscillation originates from the Little-Parks oscillations ${ }^{25}$ of the loops in the nanomesh: peaks in $T_{\mathrm{c}}$ occur at fields at which the flux through each loop is an integral multiple of $\Phi_{0}$, so fluxoid quantization is achieved by $H$ alone. Similar oscillations have been reported in superconductor micropatterned networks ${ }^{26,27}$ and films patterned with hole arrays, ${ }^{4,8}$ but only for $T \sim T_{\mathrm{c}}$ and low $H$. The oscillations of our devices extend to much higher $H$ due to the smaller length scales we have patterned. When smaller resistance criteria are used to determine $T_{\mathrm{c}}$, the oscillations at higher $H$ become less prominent, but small peak features emerge at $H$ corresponding to fractional numbers of flux quanta per unit cell, in particular for $H=$ $H_{1} / 2$. These features reflect the collective behavior of unit cells in highly ordered networks, i.e., fluxoid quantization in superunit cells. ${ }^{26,27} T_{\mathrm{c}}$ oscillations are also observed in the $34 \mathrm{~nm}$ nanomesh, but with even larger oscillation amplitude and period (Figure 2d). The observed period $H_{1}=1.65 \mathrm{~T}$ corresponds to a matching pitch of $35.4 \mathrm{~nm}$ for square networks, in agreement with the designed dimensions. Fractional matching fields are clearly observed at $H=H_{1} / 2$, and possibly at $H=1 / 3$ and $2 / 3 H_{1}$. Oscillations at $H>2 H_{1}$ are not measurable because $H_{1}$ is too high: at our base temperature, superconductivity is suppressed for $H>\sim 3 \mathrm{~T}$ (cf. Figure 2a). The observed oscillations reflect the tightly controlled structural parameters of our nanomesh films.

The critical current data are summarized in Figure 3. $J_{c}$ of the bulk film device is determined to be $3.2 \times 10^{6} \mathrm{~A} / \mathrm{cm}^{2}$ at base temperature. This value is in good agreement with previous studies on $\mathrm{Nb}$ films, ${ }^{28,29}$ but more than 1 order of magnitude lower than $J_{\text {dp }}$ (Figure 3a). Here $J_{\text {dp }}$ is taken from the pulse-current measurement data of Rusanov et al. ${ }^{30}$ We note that a $20 \mathrm{~nm}$ thick $\mathrm{Nb}$ film is measured in their study, which has a higher $T_{\mathrm{c}}$ than the $11 \mathrm{~nm}$ films we used. To compensate for this difference, we have uniformly shifted the $J_{c}-T$ curve to lower $T$ by $1.15 \mathrm{~K}$, so zero critical current is achieved at the $T_{\mathrm{c}}$ of our film device. See Supplementary Figure 3 (Supporting Information) for an alternative treatment in which $T$ is rescaled by the $T_{\mathrm{c}}$ of each device.

Drastic enhancement of $J_{c}$ is observed in the nanomesh films. For the $34 \mathrm{~nm}$ nanomesh, when reduced to the combined cross section areas of all the longitudinal NWs in the nanomesh, the measured $J_{\mathrm{cw}}$ at base temperature is 5.5 $\times 10^{7} \mathrm{~A} / \mathrm{cm}^{2}$, or more than 17 times higher than that of the starting film from which the nanomesh was fabricated. Moreover, the measured $J_{\mathrm{cw}}$ is in excellent agreement with $J_{\mathrm{dp}}$ over the entire measured temperature range, with no 

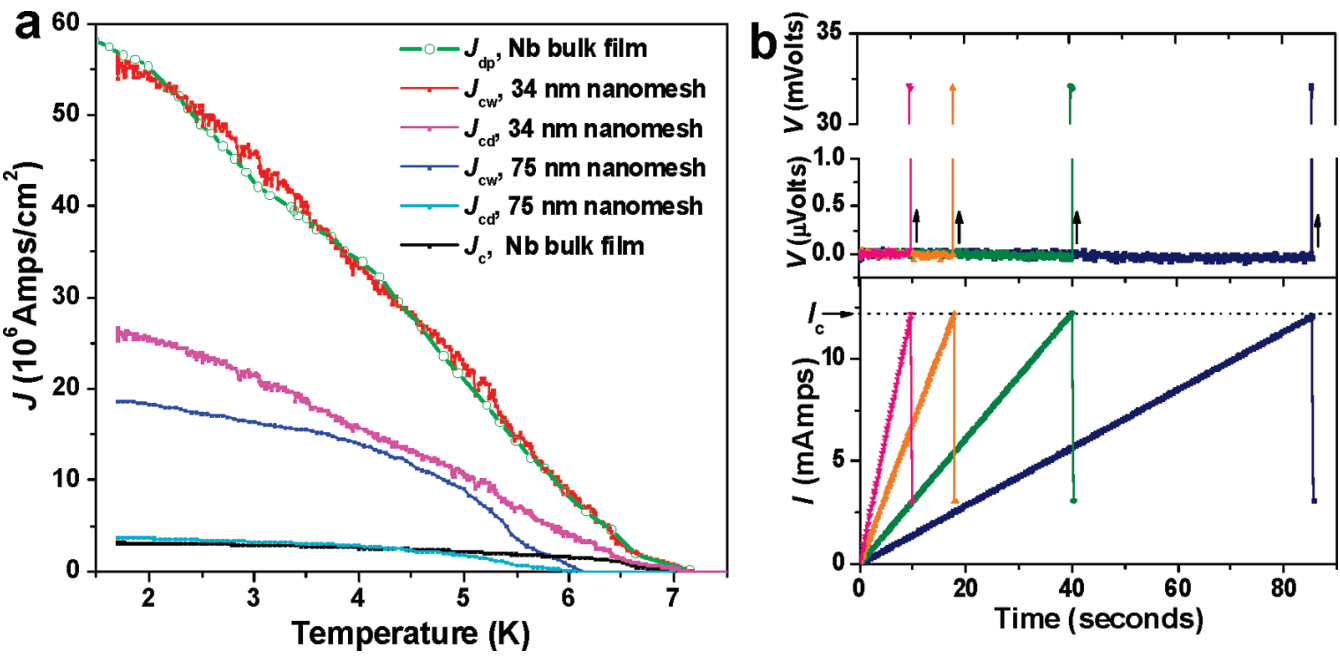

FIGURE 3. Critical current measurement. (a) Temperature-dependent critical current density $\left(J_{c}\right)$ of different devices, in comparison with the depairing current density $\left(J_{\mathrm{dp}}\right)$ of $\mathrm{Nb} . J_{\mathrm{cw}}$ is the experimental $J_{\mathrm{c}}$ when reduced to the combined cross section areas of all the longitudinal nanowires in the nanomesh. $J_{c d}$ is the experimental $J_{c}$ when reduced to the entire width of the device. (b) Critical current measurement of the $34 \mathrm{~nm}$ nanomesh at $1.7 \mathrm{~K}$, carried out at varied current scanning speeds. The current drops to a lower value at the instance when the device switches into the normal state due to the compliance of our measurement system. Lower panel: the applied current in four different scans as a function of time. Upper panel: the corresponding measured voltage as a function of time.

fitting parameters (Figure $3 \mathrm{a}$ and Supplementary Figure 3 in the Supporting Information). We emphasize that the $I_{\mathrm{c}}$ and $J_{c}$ reported here were obtained through quasi-steady dc measurements, in which a continuously applied current was monotonically increased in small steps until the device became resistive. The measured $I_{\mathrm{c}}$ was stable, independent of scanning rate, and superconductivity can be maintained for prolonged periods of time for any $I<I_{\mathrm{c}}$. As shown in Figure $3 \mathrm{~b}$, the nanomesh switches to the normal state at the same $I_{\mathrm{C}}$ for widely different current scan rates. Before this switching occurs, the device remains in the superconducting state without detectable voltage over the $\sim 30 \mathrm{nV}$ noise floor, even for $I$ very close to $I_{c}$. This is fundamentally different from the $J_{\mathrm{dp}}$ determined using pulse-current methods, ${ }^{30,31}$ in which short $(\sim 1 \mathrm{~ms})$ current pulses are employed to eliminate the effects of dissipation due to vortex motion.

The $J_{c}$ enhancement of the $75 \mathrm{~nm}$ nanomesh film is below that of the $34 \mathrm{~nm}$ nanomesh. Although the measured $J_{\mathrm{cw}}$ at base temperature $\left(1.9 \times 10^{7} \mathrm{~A} / \mathrm{cm}^{2}\right)$ is still 6-fold higher than that of the film, it is about one-third of $J_{\mathrm{dp}}$. This likely arises from multiple effects. First, $T_{\mathrm{c}}$ is reduced for this nanomesh, and so the onset of supercurrents occurs at lower $T$. When the $J_{\mathrm{dp}}-T$ curve is rescaled to compensate for this $T_{\mathrm{c}}$ difference, very good initial agreement between $J_{c w}$ and $J_{d p}$ is observed for $T>\sim 0.8 T_{\mathrm{C}}$, or within $\sim 1 \mathrm{~K}$ of $T_{\mathrm{C}}$ (Supplementary Figure 3 in the Supporting Information). At lower $T$, however, $J_{\mathrm{cw}}$ increasingly deviates from (and falls below) $J_{\mathrm{dp}}$ with decreasing $T$. This is likely because $\xi$ is large at $T \sim T_{\mathrm{C}}$, so the device behaves similar to the $34 \mathrm{~nm}$ nanomesh. At low $T, \xi$ is small and the device starts to imitate the lower $J_{c}$ of quasi-1D nanowire superconductors.

For potential applications, we note that when reduced to the entire widths of the devices, the corresponding $J_{\text {cd }}$ is reduced relative to $J_{c w}$ due to the presence of voids. For the
$34 \mathrm{~nm}$ nanomesh, $J_{\mathrm{cd}}=(16 / 34) J_{\mathrm{cw}} \sim J_{\mathrm{dp}} / 2$. At base temperature, $J_{\mathrm{cd}}=2.6 \times 10^{7} \mathrm{~A} / \mathrm{cm}^{2}$ is still more than 8-fold higher than that of the starting film. In fact, a significant enhancement of $J_{\text {cd }}$ relative to the film is achieved over the entire temperature range (Figure 3a), in particular for low $T$, where $J_{c}$ is high. This is in contrast with previous studies on micropatterned superconductors, ${ }^{4-8}$ in which $J_{c}$ enhancement is only prominent for $T$ close to $T_{\mathrm{c}}$, where $\xi$ and $\lambda$ are large and comparable with the pattern dimensions. $J_{\text {cd }}$ of the $75 \mathrm{~nm}$ nanomesh is only slightly higher than the film due to the lower $J_{\mathrm{cw}}$ and small superconductor occupation ratio (15/ 75). These results indicate that to achieve the highest possible $I_{\mathrm{c}}$ within a given device width, nanomesh films patterned at ultrahigh densities are required. More generally, these results demonstrate that the theoretical depairing limit for stable and continuous supercurrent carrying capacity within a superconductor may be achieved through appropriate structural design of the superconductor.

Acknowledgment. We thank Yue Zou for helpful discussions. This work was supported by the Department of Energy, Basic Energy Sciences.

Supporting Information Available. Description of the fabrication processes and additional discussions. This material is available free of charge via the Internet at http:// pubs.acs.org.

\section{REFERENCES AND NOTES}

(1) Tinkham, M. Introduction to Superconductivity, 2nd ed.; McGrawHill: New York, 1996.

(2) Poole, C. P. Handbook of Superconductivity; Academic Press: San Diego, CA, 2000

(3) Tsuei, C. C.; Mannhart, J.; Dimos, D. AIP Conf. Proc. 1989, 182, 194 
(4) Fiory, A. T.; Hebard, A. F.; Somekh, S. Appl. Phys. Lett. 1978, 32 73

(5) Baert, M.; Metlushko, V. V.; Jonckheere, R.; Moshchalkov, V. V.; Bruynseraede, Y. Phys. Rev. Lett. 1995, 74, 3269

(6) Yang, P.; Lieber, C. M. Science 1996, 273, 1836.

(7) Castellanos, A.; Wordenweber, R.; Ockenfuss, G.; VonderHart, A.; Keck, K. Appl. Phys. Lett. 1997, 71, 962

(8) Welp, U.; Xiao, Z. L.; Jiang, J. S.; Vlasko-Vlasov, V. K.; Bader, S. D. Crabtree, G. W.; Liang, J.; Chik, H.; Xu, J. M. Phys. Rev. B 2002, 66, 212507

(9) Likharev, K. K. Rev. Mod. Phys. 1979, 51, 101

(10) Tidecks, R. Current-Induced Nonequilibrium Phenomena in QuasiOne-Dimensional Superconductors; Springer-Verlag: Berlin, 1990

(11) Giordano, N. Phys. Rev. Lett. 1988, 61, 2137.

(12) Bezryadin, A.; Lau, C. N.; Tinkham, M. Nature 2000, 404, 971.

(13) Lau, C. N.; Markovic, N.; Bockrath, M.; Bezryadin, A.; Tinkham, M. Phys. Rev. Lett. 2001, 87, 217003

(14) Vodolazov, D. Y.; Peeters, F. M.; Piraux, L.; Matefi-Tempfli, S.; Michotte, S. Phys. Rev. Lett. 2003, 91, 157001.

(15) Tian, M. L.; Wang, J. G.; Kurtz, J. S.; Liu, Y.; Chan, M. H. W.; Mayer, T. S.; Mallouk, T. E. Phys. Rev. B 2005, 71, 104521

(16) Zgirski, M.; Riikonen, K. P.; Touboltsev, V.; Arutyunov, K. Nano Lett. 2005, 5, 1029
(17) Altomare, F.; Chang, A. M.; Melloch, M. R.; Hong, Y. G.; Tu, C. W. Phys. Rev. Lett. 2006, 97, No. 017001

(18) Xu, K.; Heath, J. R. Nano Lett. 2008, 8, 136

(19) Tinkham, M.; Free, J. U.; Lau, C. N.; Markovic, N. Phys. Rev. B 2003, 68, 134515.

(20) Rogachev, A.; Bezryadin, A. Appl. Phys. Lett. 2003, 83, 512

(21) Melosh, N. A.; Boukai, A.; Diana, F.; Gerardot, B.; Badolato, A.; Petroff, P. M.; Heath, J. R. Science 2003, 300, 112.

(22) Heath, J. R. Acc. Chem. Res. 2008, 41, 1609.

(23) Wang, D. W.; Bunimovich, Y.; Boukai, A.; Heath, J. R. Small 2007, 3, 2043.

(24) Rogachev, A.; Bollinger, A. T.; Bezryadin, A. Phys. Rev. Lett. 2005, 94, No. 017004

(25) Little, W. A.; Parks, R. D. Phys. Rev. Lett. 1962, 9, 9

(26) Pannetier, B.; Chaussy, J.; Rammal, R.; Villegier, J. C. Phys. Rev. Lett. 1984, 53, 1845.

(27) Wilks, C. W.; Bojko, R.; Chaikin, P. M. Phys. Rev. B 1991, 43, 2721

(28) Neugebauer, C. A.; Ekvall, R. A. J. Appl. Phys. 1964, 35, 547.

(29) Huebener, R. P.; Kampwirth, R. T.; Martin, R. L.; Barbee, T. W.; Zubeck, R. B. IEEE Trans. Magn. 1975, MA11, 344

(30) Rusanov, A. Y.; Hesselberth, M. B. S.; Aarts, J. Phys. Rev. B 2004, 70, No. 024510

(31) Romijn, J.; Klapwijk, T. M.; Renne, M. J.; Mooij, J. E. Phys. Rev. B $1982,26,3648$ 